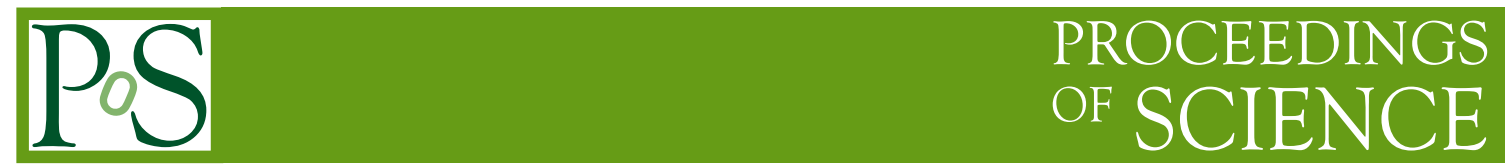

\title{
Chameleon Cosmology Near and Far
}

\author{
Amanda Weltman*广 \\ University of Cape Town, Cape Town, South Africa \\ E-mail: amanda.weltman@uct.ac.za
}

In this brief article we consider the problem of dark energy and some complementary searches and tests for alternative dark energy theories.

Frontiers of Fundamental Physics 14 - FFP14,

15-18 July 2014

Aix Marseille University (AMU) Saint-Charles Campus, Marseille

\footnotetext{
* Speaker.

${ }^{\dagger}$ My thanks to the organisers for inviting me to this meeting and to my many collaborators on all of the work discussed here.
} 


\section{Introduction}

Cosmological observations are able to pinpoint with remarkable precision details of the universe on the largest scales, while particle physics experiments probe the nature of matter on the smallest scales. These observations have left us with a portrait of the universe that is both spectacularly detailed and yet largely not well understood. One of the greatest unsolved problems of our time in fact that of vacuum energy, is the most dominant contribution to the energy density of our universe. So-called dark energy, credited with the observed accelerated expansion of the universe, makes up around $70 \%$ of the total matter budget in the universe however there is no single convincing explanation for this observation nor is there a clear pathway to distinguishing between different models through cosmological observations. If this acceleration is not caused by a cosmological constant then the most convincing explanations come in the form of phenomenological scalar field models. If a scalar field is indeed responsible for this observed acceleration it would need to be very light $m \sim H_{0}$ and evolving on cosmological scales. A priori, light fields should couple to all forms of matter with a coupling constant set by $G_{N}$ which would cause an as yet unobserved fifth force and should be observable on all scales. Thus, we are left with a puzzle as to how a scalar field can both be observable as dark energy and yet not be observed to date in all other contexts. And we are left with the grand challenge of finding such fields in a natural way perhaps through effective field theories of ultra-violet physics.

The approach described in this talk is to accept that the beast, i.e. the problem of the Dark Universe in fact (including dark matter and dark energy) may best be tackled from all sides. And that by bringing together our expertise in astrophysics, cosmology, astronomy and particle physics we may be able to design experimental and observational tests to truly figure out what is the nature of the beast. The role in cosmology of beyond the standard model physics is perhaps not quite fully realised though there has been enormous progress in the last five to ten years. Indeed the remarkable realisation is that there are many relatively low cost ways to test our ideas and putting these together can create a rather high impact physical understanding of our universe through low intensity and low cost physics. The particular problem tackled in this brief article will be that of dark energy with chameleon gravity as the particular approach.

\section{Chameleon Gravity}

A solution to the puzzle of having gravitationally coupled scalar fields was presented in $[1,2,3]$ with so-called chameleon fields. Chameleon fields are a compelling dark energy candidate; they couple to all Standard Model particles without violating any known laws of physics or experiments done to date. They are testable complementary ways to the standard observational cosmology techniques, and so provide a new window into dark energy through laboratory and astrophysical tests and space tests of gravity. Such a coupling, if detected, could reveal the nature of dark energy and may point the way to the development of an ultraviolet completion of gravity.

A canonical scalar field is the simplest dynamical extension of the Standard Model that could explain dark energy. Scalar field dark energy models typically require a self interaction, resulting in a 
nonlinear equation of motion $[4,5]$. Such a self interaction, with a matter coupling, gives the scalar field a large effective mass in regions of high matter density [1,2]. A scalar field that is massive locally mediates a short-range fifth force that is difficult to detect and effectively hides depending on its environment - earning it the name "chameleon field." Furthermore, the massive chameleon field is sourced only by the thin shell of matter on the outer surface of a dense extended object. These nonlinear effects serve to screen fifth forces, making them more difficult to detect in certain environments.

A chameleon field couples to dark matter and all matter types, in principle with independent strengths. At the classical level, a chameleon field is not required (nor forbidden) to couple to photons. However, when quantum corrections are included, a photon coupling about three orders of magnitude smaller than the matter coupling is typically generated [6]. The lowest order chameleonphoton interaction couples the chameleon field to the square of the photon field strength tensor thus in a background electromagnetic field, photons can oscillate into chameleon particles and back.

The mass of chameleon fields produced will depend on the environmental energy density as well as the local electromagnetic field strength. This provides for great potential for experiments on Earth, in space, and through astrophysical observations. Several astrophysical puzzles could also be explained by chameleons, e.g., [8]. Their coupling to photons, combined with their light masses in certain environments, allows chameleons to be produced with intense beams of photons, electrons, or protons and detected with sensitive equipment.

\subsection{Ingredients}

In [3] an action of the following form is proposed for Chameleon fields coupled to matter and photons;

$$
S=\int d^{4} x \sqrt{-g}\left(\frac{1}{2 M_{p l}^{2}} R-\partial_{\mu} \phi \partial^{\mu} \phi-V(\phi)\right)-\frac{e^{\phi / M_{\gamma}}}{4} F^{\mu v} F_{\mu v}+S_{m}\left(e^{2 \phi / M_{m}^{i}} g_{\mu v}, \psi_{m}^{i}\right),
$$

where $S_{m}$ is the action for matter and in general the chameleon field, $\phi$ can couple differently to different matter types $\psi_{i}$, and $V(\phi)$ is the chameleon self interaction. For simplicity here we will consider a universal coupling to matter defined by $\beta_{m}=M_{\mathrm{Pl}} / M_{m}$ while allowing for a different coupling to electromagnetism, $\beta_{\gamma}=M_{\mathrm{Pl}} / M_{\gamma}$, through the electromagnetic field strength tensor $F_{\mu \nu}$.

The non-trivial coupling to matter and the electromagnetic field induces an effective potential

$$
V_{\mathrm{eff}}(\phi, \vec{x})=V(\phi)+e^{\beta_{m} \phi / M_{\mathrm{Pl}}} \rho_{m}(\vec{x})+e^{\beta_{\gamma} \phi / M_{\mathrm{Pl}}} \rho_{\gamma}(\vec{x}),
$$

where we have defined the effective electromagnetic field density $\rho_{\gamma}=\frac{1}{2}\left(\left.\left|\vec{B}^{2}-\right| \vec{E}\right|^{2}\right)$ rather than the energy density. An essential insight of chameleon models is noticing that the presence of matter and electromagnetic fields induces a minimum $\phi_{\min }$ in $V_{\text {eff }}$ where $V$ can be a monotonic function. The dependence of this minimum on the background matter and electromagnetic fields causes the 
effective mass of the chameleon field to change in response to its environment. In turn we find varied chameleon phenomenology depending on the experimental setup and hence the environment.

We can see explicitly that for an exponential potential, the effective mass of the field $\phi$ is dependent on the local density of matter and electromagnetic fields,

$$
V(\phi)=\Lambda^{4} \exp \left(\frac{\Lambda^{n}}{\phi^{n}}\right), \quad \phi_{\min } \approx\left(\frac{n M_{\mathrm{Pl}} \Lambda^{n+4}}{\beta_{\mathrm{m}} \rho_{m}+\beta_{\gamma} \rho_{g}}\right)^{\frac{1}{n+1}} \text { and } m_{\phi}^{2} \approx \frac{(n+1)}{\left(n \Lambda^{n+4}\right)^{\frac{1}{n+1}}}\left(\beta_{m} \rho_{m}+\beta_{\gamma} \rho_{\gamma}\right)^{\frac{n+2}{n+1}}
$$

where the next to leading order terms are suppressed by factors of $\beta_{i} \phi / M_{\mathrm{Pl}} \ll 1$.

The chameleon dark energy parameter space is complicated but under some assumptions general constraints can be given. With the caveat that all matter couplings are the same but not equal to the photon coupling, and the assumption of a specific chameleon potential, $V(\phi)=M_{\Lambda}^{4}(1+$ $\left.M_{\Lambda}^{n} / \phi^{n}\right)$ in which we set the scale $M_{\Lambda}=2.4 \times 10^{-3} \mathrm{eV}$ to the observed dark energy density and, for concreteness, $n=1$, our constraints and forecasts are provided by Figure 1. Current constraints (solid regions) and forecasts (curves) are discussed below.

\section{Current Constraints and Forecasts}

\subsection{Current laboratory constraints}

Chameleon dark energy is constrained in the lab in two concretely different types of experiments. Fifth force searches that probe matter couplings and direct photon coupling experiments, both of which are shown as shaded regions in Figure 1. Gravitation-strength fifth forces can be measured directly between two macroscopic objects, such as the source and test masses in a torsion pendulum. Currently the shortest-range torsion pendulum constraints on gravitation-strength forces come from the Eöt-Wash experiment [9]. An ultracold gas of neutrons can be used as a fifth force experiment as explained in [10]. If the neutrons feel a fifth force from the experimental apparatus comparable to the gravitational force of the Earth, then the energy splittings will be altered. The Grenoble experiment measures these energy splittings at the $\sim 10 \%$ level, excluding very strong matter couplings $\beta_{m} \gtrsim 10^{11}$.

Dark energy may couple to the electroweak sector in addition to matter. Such a coupling would allow photons propagating through a magnetic field to oscillate into chameleons which can then be trapped inside a chamber. An "afterglow experiment" produces dark energy particles through oscillation and then switches off the photon source, allowing the population of trapped dark energy particles to regenerate photons to later emerge as an afterglow. Current afterglow constraints from the CHASE (CHameleon Afterglow SEarch) experiment exclude photon couplings $10^{11} \lesssim \beta_{\gamma} \lesssim$ $10^{16}$ for $\beta_{m} \gtrsim 10^{4}$, as shown in Fig. 1 for an inverse-power-law chameleon potential $[11,12,13]$. At yet higher photon couplings the trapped dark energy particles regenerate photons too quickly for CHASE to detect them. However, collider experiments can exclude such models, by constraining chameleon loop corrections to precision electroweak observables [14]. 


\begin{tabular}{|l||c|c|}
\hline Experiment & Type & Couplings excluded \\
\hline \hline Eöt-Wash & torsion pendulum & $0.01 \lesssim \beta \lesssim 10$ \\
\hline Lamoreaux & Casimir & $\beta \gtrsim 10^{5}\left(\phi^{4}\right)$ \\
\hline Grenoble & bouncing neutron & $\beta \gtrsim 10^{11}$ \\
\hline GRANIT & bouncing neutron & forecast: $\beta \gtrsim 10^{8}$ \\
\hline NIST & neutron interferometry & forecast: $\beta \gtrsim 10^{7}$ \\
\hline CHASE & afterglow & $10^{11} \lesssim \beta_{\gamma} \lesssim 10^{16}$ subject to $10^{4} \lesssim \beta_{m} \lesssim 10^{13}$ \\
\hline ADMX & microwave cavity & $m_{\text {eff }}=1.952 \mu \mathrm{eV}, 10^{9} \lesssim \beta_{\gamma} \lesssim 10^{15}$ \\
\hline CAST & helioscope & forecast: $\beta_{m} \lesssim 10^{9}, \beta_{\gamma}>10^{10}$ \\
\hline
\end{tabular}

Table 1: Laboratory tests of dark energy. Approximate constraints on chameleon models with potential $V(\phi)=M_{\Lambda}^{4}\left(1+M_{\Lambda} / \phi\right)$ and $M_{\Lambda}=2.4 \times 10^{-3} \mathrm{eV}$ (unless otherwise noted). From [15]

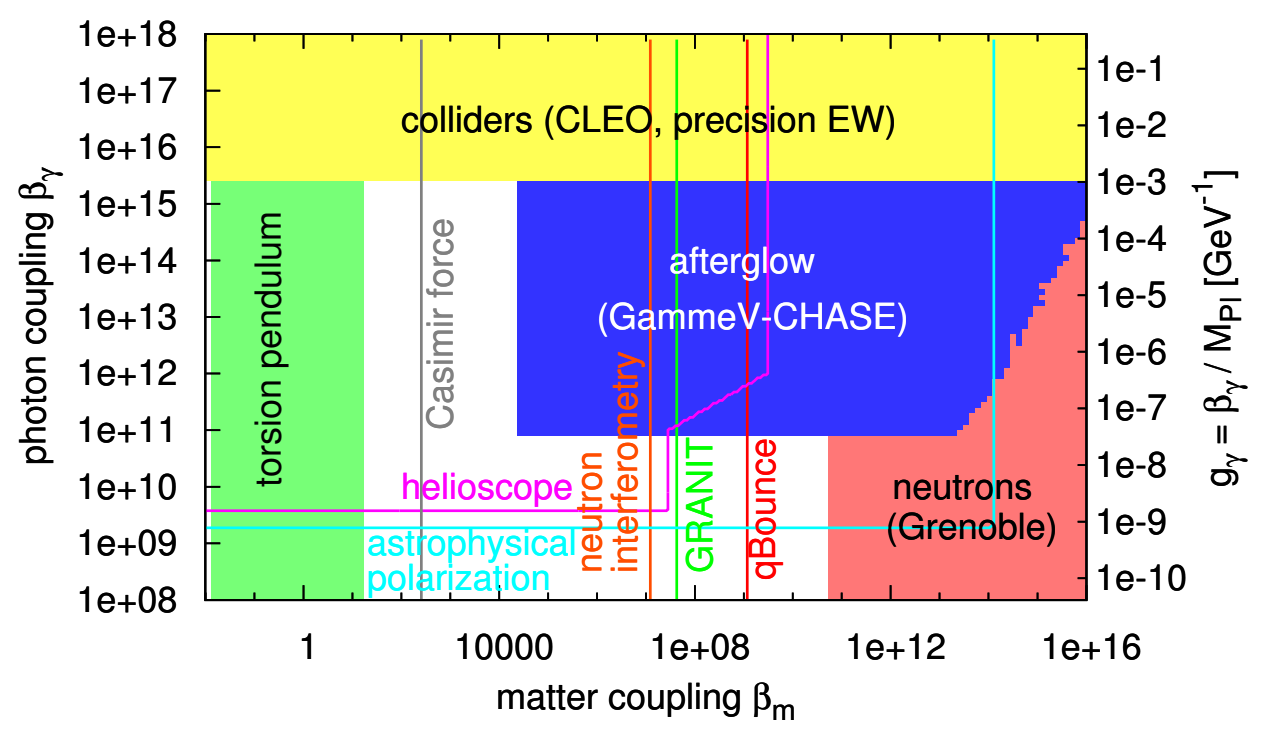

Figure 1: Constraints on the matter and photons couplings for a chameleon dark energy model with $V(\phi)=$ $M_{\Lambda}^{4}\left(1+M_{\Lambda} / \phi\right)$. Current constraints are shown as shaded regions, while forecasts are shown as solid lines. From [16]

\subsection{Forecasts for Terrestrial experiments}

Proposed experiments promise to improve constraints on chameleon dark energy by orders of magnitude over the next several years. Figure 1 summarizes forecasts and preliminary constraints, shown as solid lines. The next-generation Eöt-Wash experiment, currently under way, will have an increased force sensitivity and probe smaller distances. This will allow it to detect or exclude a large class of chameleon models with well-controlled quantum corrections [17, 18]. Improvements to fifth force measurements using neutrons should improve constraints on the chameleon-matter coupling considerably. Also proposed is a neutron interferometry experiment at NIST, which should be competitive with the bouncing neutron experiments. A neutron interferometer splits a neutron beam and sends the two through two different chambers, one containing a dense gas which suppresses 
chameleon field perturbations, and the other a vacuum chamber in which scalar field gradients are large. These gradients will retard the neutron beam passing through the vacuum chamber, resulting in a phase shift which varies nonlinearly with the gas pressure. Potentially more powerful are the next-generation Casimir force experiments [19]. However, these currently suffer from systematic uncertainties including the proper calculation of thermal corrections to the Casimir effect. The forecasts shown require that the total uncertainty in the Casimir force be reduced below $1 \%$ at distances of $5-10 \mu \mathrm{m}$.

Other planned experiments search for photon-coupled chameleons. Afterglow experiments have been proposed at JLab and the Tore Supra tokamak, while a microwave cavity-based afterglow experiment is under way at Yale. Since forecasts for these experiments are not available for the chameleon potential assumed in Fig. 1, we are unable to include them in the figure. However, the JLab and Tore Supra experiments are expected to fill in some of the gap between CHASE and torsion pendulum experiments, while the microwave cavity search is a precision experiment capable of targeting a model with a specific mass in response to hints from an afterglow experiment. Yet another type of experiment is the helioscope, which uses a high magnetic field to regenerate photons from scalar particles produced in the Sun [20]. Since such particles do not need to be trapped prior to detection, helioscope forecasts extend down to arbitrarily low matter couplings. One proposed helioscope (IAXO) adds an X-ray mirror to the CAST axion helioscope at CERN in order to increase its chameleon collecting area; forecasts for this experiment are shown [21]

\subsection{Tests using Astrophysical Observation}

Perhaps most promising for future chameleon searches is the rich phenomenology of unique astrophysical signatures offered by chameleons. Combining data from astrophysical observations with laboratory experimental data will allow us to constrain chameleon models to unprecedented levels. Below we review some of the more intriguing astrophysical signatures predicted in chameleon models. One benefit of observational tests of chameleons is that these observations may be performed complementarily with observations taken for reasons not related to chameleon gravity.

The screening mechanism from chameleons has significant consequences for structure formation. These modifications include an earlier collapse of density perturbations compared to the prediction from $\Lambda \mathrm{CDM}$ and clumpier dark matter halos [22] and the critical density required for collapse depends on the comoving size of the inhomogeneity itself [23]. Galactic satellite orbits become modified based on the size of the satellite due to a backreaction from the satellite causing a velocity difference of up to $10 \%$ near the thin shell [24].

Another prediction of chameleon gravity is that in unscreened environments, (such as voids) stellar structure is modified, most notably in the red giant branch of stars. The authors of [26] found that chameleons affect the size and temperature of red giant stars where they tend to be smaller ( $\sim 10 \%)$, and hotter ( $\sim 100$ s of Kelvins). Also, observations of circularly polarized starlight in the wavelength range $1-10^{3}$ could be a strong indication of chameleon-photon mixing [27].

Astrophysical tests of chameleons in $f(R)$ theories may be parameterized by how efficiently bodies self-screen $(\chi)$ and the strength of the fifth force $(\alpha)$ [28]. For the case of chameleon $f(R)$ gravity, $\chi \equiv d f / d R$ is measured at present time. The additional force is parametrized by rescaling Newton's constant $G \rightarrow G(1+\alpha)$ for unscreened objects and $G(r) \rightarrow G\left[1+\alpha\left(1-M\left(r_{s}\right) / M(r)\right)\right]$ 
for partially screened objects. Fifth forces are screened at radii $r<r_{s}$, unscreened for radii $r_{s}<$ $r$, and $M(r)$ is the mass contained within a shell at radius $r$. For an object to be unscreened, $\Phi_{N} \ll \chi$ where $\Phi_{N}$ is the Newtonian potential. The Sun and Milky Way (coincidentally) possess a similar gravitational potential: $\Phi_{\odot} \sim 2 \times 10^{-6}$ and $\Phi_{M W} \sim 10^{-6}$. Stars in the tip of the red giant branch of the HR diagram and Cepheid variables have gravitational potentials $\Phi_{N} \sim 10^{-7}$. These stars will have their outer layers unscreened provided they reside in smaller galaxies in a shallow gravitational potential. For fifth forces of a strength described by $\alpha=1 / 3$, values of $\chi$ greater than $5 \times 10^{-7}$ may be ruled out at $95 \%$ confidence. This upper bound is moderately lower for fifth force strength defined by $\alpha=1$, where values of $\chi$ greater than $1 \times 10^{-7}$ may be ruled out at a $95 \%$ confidence level [28] (also see Fig.(5) of [28]). These constraints on $\chi$ and $\alpha$ from local universe observations are stronger than current cosmological constraints on chameleon fifth forces [29]-[31] which typically give an upper limit not less than $\chi \sim 10^{-6}$.

\subsection{Space tests of Gravity}

Remarkably, the original predictions of signatures in space for chameleon models would still be the most striking [1,2]. The proposed experiments discussed there have not yet taken place. However, the MicroSCOPE [32] mission and STE-QUEST [33] are future satellite experiments that hold the promise of testing these theories in a way complementary to the terrestrial and astrophysical methods discussed here. The expected signatures are large and for example an $\mathscr{O}(1)$ observed difference in Newton's constant for unscreened objects would be a smoking gun for these models. There is hope that with MicroSCOPE launching in the coming year that we will see some new exciting results.

\section{Conclusions}

The outlook is very bright for chameleon gravity. There is still a lot of work to be done but the progress has been very promising on all fronts, from astrophysical to laboratory. The diversified approach to this problem appears to be paying off and in the long run we hope to see these fields either fully constrained or convincely observed.

\section{References}

[1] J. Khoury and A. Weltman. Phys. Rev. Lett., 93, 2004. 171104.

[2] J. Khoury and A. Weltman. Phys. Rev. D, 69, 2004. 044026.

[3] P Brax, C. van de Bruck, A. C. Davis, J. Khoury, A. Weltman, Aug 2004. 31pp. Published in Phys.Rev.D70:123518,2004. e-Print: astro-ph/0408415

[4] P. J. E. Peebles and B. Ratra. Ap. J. Lett., 325:17, 1988.

[5] B. Ratra and P. J. E. Peebles. Phys. Rev. D, 37(12):3406, 1988.

[6] P. Brax, C. Burrage, A.-C. Davis, D. Seery, and A. Weltman. Phys. Rev. D, 81:103524, 2010. e-Print arXiv:0911.1267.

[7] E. G. Adelberger et al. Phys. Rev. Lett., 98:131104, 2007. e-Print arXiv:hep-ph/0611223. 
[8] L. Hui, A. Nicolis and C. Stubbs, "Equivalence Principle Implications of Modified Gravity Models," Phys. Rev. D 80 (2009) 104002 [arXiv:0905.2966 [astro-ph.CO]].

[9] D. J. Kapner, T. S. Cook, E. G. Adelberger, J. H. Gundlach, B. R. Heckel, C. D. Hoyle, and H. E. Swanson. Phys. Rev. Lett., 98:021101, 2007. e-Print arXiv:hep-ph/0611184.

[10] P. Brax and G. Pignol. Phys. Rev. Lett., 107:111301, 2011.

[11] J. H. Steffen et al. Phys. Rev. Lett., 105:261803, 2010. ePrint: arXiv:1010.0988.

[12] A. Upadhye, J. H. Steffen, and A. Weltman. Phys. Rev. D, 81:015013, 2010.

[13] A. Upadhye, J. H. Steffen, and A. S. Chou. Phys. Rev. D, 86:035006, 2012.

[14] P. Brax, C. Burrage, A.-C. Davis, D. Seery, and A. Weltman. JHEP, 0909:128, 2009. e-print arXiv:0904.3002.

[15] B. Jain, A. Joyce, R. Thompson, A. Upadhye, J. Battat, P. Brax, A. C. Davis and C. de Rham et al., arXiv:1309.5389 [astro-ph.CO].

[16] R. Essig, J. A. Jaros, W. Wester, P. H. Adrian, S. Andreas, T. Averett, O. Baker and B. Batell et al., arXiv:1311.0029 [hep-ph].

[17] A. Upadhye, W. Hu, and J. Khoury. Phys. Rev. Lett, 109:041301, 2012.

[18] A. Upadhye. Phys. Rev. D, 86:102003, 2012. e-Print: arXiv:1209.0211.

[19] P. Brax, C. van de Bruck, A. C. Davis, D. F. Mota, and D. J. Shaw. Phys. Rev. D, 76:124034, 2007. e-Print arXiv:0709.2075.

[20] P. Brax, A. Lindner and K. Zioutas, Phys. Rev. D 85 (2012) 043014 [arXiv:1110.2583 [hep-ph]].

[21] E. Armengaud, F. T. Avignone, M. Betz, P. Brax, P. Brun, G. Cantatore, J. M. Carmona and G. P. Carosi et al., JINST 9, T05002 (2014) [arXiv:1401.3233 [physics.ins-det]].

[22] P. Brax, C. van de Bruck, A. -C. Davis and A. M. Green, Phys. Lett. B 633 (2006) 441 [astro-ph/0509878].

[23] P. .Brax, R. Rosenfeld and D. A. Steer, JCAP 1008 (2010) 033 [arXiv:1005.2051 [astro-ph.CO]].

[24] R. Pourhasan, N. Afshordi, R. B. Mann and A. C. Davis, JCAP 1112 (2011) 005 [arXiv:1109.0538 [astro-ph.CO]].

[25] C. Burrage, Phys. Rev. D 77 (2008) 043009 [arXiv:0711.2966 [astro-ph]].

[26] P. Chang and L. Hui, Astrophys. J. 732 (2011) 25 [arXiv:1011.4107 [astro-ph.CO]].

[27] C. Burrage, A. -C. Davis and D. J. Shaw, Phys. Rev. D 79 (2009) 044028 [arXiv:0809.1763 [astro-ph]].

[28] B. Jain, V. Vikram and J. Sakstein, arXiv:1204.6044 [astro-ph.CO].

[29] F. Schmidt, A. Vikhlinin and W. Hu, Phys. Rev. D 80 (2009) 083505 [arXiv:0908.2457 [astro-ph.CO]].

[30] L. Lombriser, A. Slosar, U. Seljak and W. Hu, Phys. Rev. D 85 (2012) 124038 [arXiv:1003.3009 [astro-ph.CO]].

[31] L. Lombriser, K. Koyama, G. -B. Zhao and B. Li, Phys. Rev. D 85 (2012) 124054 [arXiv:1203.5125 [astro-ph.CO]].

[32] http://microscope.onera.fr/

[33] http://sci.esa.int/science-e/www/area/index/cfm?fareaid=127 